a sustained low prevalence of TDR to the PIs, recent accumulation of resistance associated to the NRTIs and reduction to NNRTIs over the years. The time trend of TDR observed, seem to reflect changes in antiretroviral therapy in Brazil over time. HIV-1 subtype B was the most prevalent in the study, but the increasing prevalence of subtype $\mathrm{C}$ and the identification of others non-B and recombinants infections, suggest the recent introduction and spreading of these viruses, respectively south Brazil and African countries in Rio de Janeiro.

Support: Oswaldo Cruz Foundation-IOC/FIOCRUZ, Brazilian Ministry of Health (DDAHV-CQV/MS), Pan-American Health Organization-PAHO and World Health Organization-WHO

\section{P2.23 HEPATOTOXICITY AND ANAEMIA CO-MORBIDITY IN TREATED AIDS PATIENTS IN FUNDONG SUB DIVISION IN THE NORTHWEST REGION OF CAMEROON}

${ }^{1}$ Lem Edith Abongwa, ${ }^{2}$ Anthony Kebira Nyamache, ${ }^{1}$ Nantia Akono, ${ }^{1}$ Stanley Sunjo, ${ }^{1}$ Vera Nange, ${ }^{1}$ Hebert Balan, ${ }^{3}$ Fokunang Charles, ${ }^{2}$ Paul Okemo, ${ }^{3}$ Judith Torimiro. ${ }^{1}$ University of Bamenda, Faculty of Science, Department of Biological Sciences, P.O. Box 39, Bambili, Bamenda - Cameroon; ${ }^{2}$ School of Pure and Applied Sciences, Department of Microbiology, Kenyatta University, P.O. Box 43, Nairobi, Kenya; ${ }^{3}$ Faculty of Medicine and Biomedical Sciences, University of Yaounde I, Yaounde, Yaounde - Cameroon

\subsection{6/sextrans-2017-053264.199}

Introduction Hepatotoxicity and anaemia are relevant adverse effects of ART and can cause interruption of therapy and death. However, there is dearth of information on hepatotoxicity and anaemia co-morbidity especially in rural areas. The aim of the study was to identify the prevalence of Hepatotoxicity and Anaemia co-morbidity among HIV treated patients.

Methods A total of 150 drug naïve patients visiting the day hospital in Fundong District Hospital were recruited into the study from January-March 2015 and follow up for 18 months. Baseline and 18 months levels of CD4 counts, alanine transaminase(ALT), and aspartate transaminase(AST) and Haemoglobin concentration $(\mathrm{Hb})$ were determined. HIV was diagnosed using Alere determine HIV rapid test kit and Bioline or Oral Quick test kit for the confirmatory test. CD4 counts were determined using the Alere Pima CD4 cartridge machine. $\mathrm{Hb}$, ALT and AST counts were determined by colometric enzymatic reaction using the urit 3300 machine and classified based on age and sex.

Results The majority of patients were female $115(76.7 \%)$ and belonged to the $<30$ years age range 48 (32\%). The prevalence of anaemia decreased from $86(57.3 \%)$ to $69(45.6 \%)$ at the end of the study period. In all 46 (30.7\%) patients had hepatotoxicity and anaemia co-morbidity which was higher in the age group $<30$ years $30(41.7 \%)$ and in female 37 $(32.2 \%)$. A total of $1(0.7 \%)$ and $10(6.7 \%)$ patients develop severe hepatotoxicity using ALT and AST respectively. The prevalence of hepatotoxicity was higher in male $31.4 \%$ and $62.9 \%)$ and in the age group 30-39 years $(29.5 \%$ and $68.2 \%)$ for ALT and AST, respectively. The prevalence of anaemia and elevated AST and ALT were higher in persons with CD4 <200 cells $/ \mu$ l. There was a significant correlation between $\mathrm{CD} 4$ and $\mathrm{Hb}(\mathrm{r}=0.193), \mathrm{CD} 4$ and $\operatorname{ALT}(\mathrm{r}=-0.149)$ and CD4 and AST(r=-0.193).

Conclusion Hepatotoxicity especially Grades 1-2 and not anaemia is a significant adverse effect of ART upon time.

\section{$\mathrm{P} 2.24$}

AWARENESS AND INTEREST IN PRE-EXPOSURE PROPHYLAXIS (PREP) AMONG PATIENTS RECEIVING SERVICES AT A PUBLIC SEXUALLY TRANSMITTED DISEASES (STD) CLINIC IN A HIGH PREVALENCE URBAN SETTING

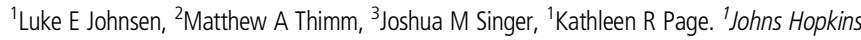
University School of Medicine, Baltimore City Health Department, Baltimore, Md, USA; ${ }^{2}$ Johns Hopkins University School of Medicine, Baltimore, Md, USA; ${ }^{3}$ University of Maryland, College Park, MD, USA

\subsection{6/sextrans-2017-053264.200}

Introduction HIV rates in Baltimore City are among the highest in the United States, with the majority of new infections attributed to male-to-male sexual contact. In 2016, the Baltimore City Health Department (BCHD) implemented a PreExposure Prophylaxis (PrEP) program at their sexually transmitted diseases (STD) clinics. We assessed awareness, interest, and sources of knowledge about PrEP among patients served at these clinics.

Methods We surveyed a convenience sample of 1675 patients who attended BCHD clinics between 4/12/2016 and 10/3/ 2016. Participants were provided a self-administered survey that assessed awareness, interest, and sources of knowledge about PrEP, as well as age, race, sex, and sexual preference. Univariate and multivariable logistic regression analysis examined predictors of awareness and interest. Data collection will continue in 2017 to determine if awareness and interest change over time.

Results Mean age of participants was $32.1 \quad(\mathrm{SD}=11.8)$ and $61.0 \%$ were male. $91.1 \%$ of participants self-identified as African American, 5.7\% as white, and 3.2\% as "Other." $20.0 \%$ of the participants were aware of PrEP and $42.2 \%$ were interested in PrEP. Less than 1\% of all participants and $9.2 \%$ of men who have sex with men (MSM) reported current PrEP use. White participants were more likely to be aware of PrEP than African Americans $(O R=1.76, p=0.026)$, but there was no significant difference in interest between these groups $(p=0.122)$. By univariate analysis men were more likely to be aware of PrEP than women $(\mathrm{OR}=1.38, \mathrm{p}=0.013)$, but multivariable analysis eliminated this significant difference $(p=0.081)$. MSM were significantly more likely to be aware $(\mathrm{OR}=14.29, \mathrm{p}<0.001)$ and interested $(\mathrm{OR}=1.59, \mathrm{p}=0.024)$ in PrEP. Sources of knowledge included healthcare providers (40.0\%), friends (24.7\%), and television (16.4\%).

Conclusion MSM receiving care at the Baltimore City STD Clinics are significantly more likely to be aware and interested in PrEP, but few are taking PrEP, highlighting a need to improve access and promote uptake in this high risk population.

\section{P2.25 PULMONARY RHODOCOCCOSIS WITH BACTEREMIA AS AN AIDS DEFINING INFECTION: CASE REPORT}

Marcos Davi Gomes de Sousa, Juliana Neves Ferreira de Oliveira, Ofelio Alberto Manuel Andrea Varon, Maria Cristina da Silva Lourenço, Erica Aparecida dos Santos, Cristiane Lamas. National Institute of Infectology Evandro Chagas, Rio de Janeiro - RJ, Brazil

\subsection{6/sextrans-2017-053264.201}

Introduction In endemic countries for tuberculosis (TB), HIVTB coinfection is very common. However, other bacteria such as Rhodococcus produce similar clinical and radiological 